
absorption and production during walking

3 Veronica Farinelli ${ }^{1,2}$, Ladan Hosseinzadeh ${ }^{1}$, Chiara Palmisano ${ }^{1,3}$, Carlo Frigo ${ }^{1}$

$4 \quad{ }^{1}$ Movement Biomechanics and Motor Control Lab, DEIB, Politecnico di Milano, Milan, Italy

52 Human Physiology Section of the De.P.T., Università degli Studi di Milano, Milan, Italy

$6 \quad{ }^{3}$ University Hospital and Julius-Maximilian-University, Wuerzburg, Germany

7 Corresponding author

8 Veronica Farinelli

$9 \quad$ veronica.farinelli@mail.polimi.it

\title{
10 Acknowledgements
}

11 The authors would like to thank Prof. Paolo Cavallari (DePT, University of Milan) for making available 12 his laboratory facilities. Chiara Palmisano was supported by a grant of the German Excellence 13 Initiative to the Graduate School of Life Sciences, University of Wuerzburg.

14 This work has been partially supported by the Lombardy Region grant: Linea R\&S per Aggregazione Project ID 147630 CUP E17H16001450009, and by Fondazione Mariani for Child 16 Neurology. 
Abstract

Background: Power and energy at the ankle joint during gait are usually computed considering the foot as a rigid body. The foot is instead a deformable structure and can absorb and produce energy by pronation/supination, foot arch deformation and other intrinsic movements. Research question: Is it feasible to improve the foot power and energy estimation during gait with a simple gait analysis protocol?

Materials and Methods: The power exchanged between the foot and the shank was computed as the sum of rotational and translational power, intrinsically considering the foot deformation ("Deformable Foot method", DF). By this method the only shank movements and ground reaction forces need to be analysed. Eighteen healthy subjects were evaluated while walking barefoot and shod at different velocities. We then compared the obtained results with those obtained by the conventional power and energy calculation method ("Ankle Joint method", AJ).

Results: The DF method showed a consistent negative peak of power absorption during the load acceptance $(-1.16 \pm 0.47 \mathrm{~W} / \mathrm{Kg}$ barefoot, $-1.08 \pm 0.44 \mathrm{~W} / \mathrm{Kg}$ shod), barely visible with the AJ method ($0.23 \pm 0.09 \mathrm{~W} / \mathrm{Kg}$ barefoot, $-0.30 \pm 0.09 \mathrm{~W} / \mathrm{Kg}$ shod). The maximum power production calculated with the DF method (2.44 $\pm 0.56 \mathrm{~W} / \mathrm{Kg}$ barefoot, $2.49 \pm 0.57 \mathrm{~W} / \mathrm{Kg}$ shod) was significantly lower than to the one calculated with the AJ method ( $3.15 \pm 0.68 \mathrm{~W} / \mathrm{Kg}$ barefoot, $3.09 \pm 0.69 \mathrm{~W} / \mathrm{Kg}$ shod). Similarly, the final energy values, the energy absorbed and produced were different between the two methods. Significance: Neglecting the foot deformations during gait leads to underestimate power absorption and overestimate power production. The DF method does not require a complex gait analysis protocol and can provide important information about the internal structure of the foot, thus improving physiological and clinical assessment.

Keywords: walking energy, deformable foot, gait analysis, ankle joint 


\section{Introduction}

Foot is not a rigid body. It is a complex structure composed by 23 bones connected by ligaments and subjected to the action of several muscles. When loaded by body weight during walking, the structure undergoes deformations consisting of rearfoot pronation/supination, lowering of the medial arch, changing of the widening between first and fifth metatarsi, flexion of the phalanges in the late stance phase, and many other intrinsic movements occurring both in the stance and in the swing phase. A nice description of these movements can be obtained by using foot specific movement analysis protocols. In particular, the so-called Heidelberg foot model [1] aims at the analysis of those intrinsic movement that have a clinical relevance and can be measured without any arbitrary assumption about internal rotational axes. Other models, like the Oxford foot model [2] consider the foot as composed of three segments: hindfoot, forefoot and hallux, and aims at providing the relative rotations between them. Although the number of proposed foot models is relatively high (comments about this can be found in [3]) seldom these models are implemented in current gait analysis. The reason can be related to the complex and delicate procedure of markers placement required by these models, a critical aspect that can strongly affect the results. In most gait analysis studies, a very simplified representation of the foot is adopted, consisting in a single rigid body connected to the shank by a rotational hinge. This use has been consolidated in several papers and textbooks [4-9]. These models can provide a general description of dorsi-plantar flexion of the forefoot and, eventually, of its eversion-inversion, but they are unsuitable to capture the rearfoot movements and establish how much energy is absorbed and produced by the effect of foot deformation. This problem has been faced very thoroughly in recent publications [10-12], in which the authors compare different approaches for ankle and foot power computation during walking and warn against modelling the foot as a single rigid-body. According to these studies, the ankle joint power computed as the product of joint moment and angular velocity would underestimate the amount of energy absorbed by the foot during load acceptance and overestimates the muscle energy production in the push-off phase. They proposed a new method for power and energy estimation which intrinsically considers the role of foot deformation in energy absorption and production. Since a correct evaluation of energy and power can be extremely important for the clinical interpretation of data obtained from gait analysis, we investigated the possibility to adopt the proposed method of energy computation in a clinical setting, comparing its performance with the results obtained by modelling the foot as a rigid body. The objective was to achieve a reasonable accuracy of the energy estimation without increasing the complexity of the protocol. In the present study, we show that this is feasible and worthwhile. Indeed, the results show that the foot power and energy profiles obtained by the two methods present relevant qualitative and quantitative differences, both in barefoot and in shod walking conditions.

\section{Methods}

Power and energy calculation

In order to account for energy transfer from the ground to the shank during foot support, the formula proposed by the mentioned authors $[10,11]$ is:

$$
P_{g r-s h}=F_{G R} \cdot\left(V_{A J}+\omega_{s h} \times r_{C O P-A J}\right)+M_{\text {free }} \times \omega_{s h}
$$


Where $P_{g r-s h}$ is the power transmitted to the shank; $F_{G R}$ is the ground reaction force; $V_{A J}$ is the linear velocity of a point at the basis of the shank (the centre of the ankle joint); $\omega_{\text {sh }}$ is the rotational velocity of the shank segment; $r_{C O P-A J}$ is the vector from the centre of pressure COP and the ankle joint centre; $M_{\text {free }}$ is the free moment of ground reaction (the only vertical component of this moment is different from zero). The formula can be rearranged as:

$$
P_{g r-s h}=F_{G R F} \cdot V_{A J}+F_{G R} \cdot\left(\omega_{s h} \times r_{C O P-A J}\right)+M_{\text {free }} \cdot \omega_{s h}
$$

Which is equivalent to:

$$
P_{g r-s h}=F_{G R} \cdot V_{A J}+\left(r_{C O P-A J} \times F_{G R} \cdot \omega_{s h}\right)+M_{\text {free }} \cdot \omega_{s h}
$$

And thus, finally:

$$
P_{g r-s h}=F_{G R} \cdot V_{A J}+\left(M_{G R-A J}+M_{f r e e}\right) \cdot \omega_{s h}
$$

Where $M_{G R-A}=r_{C O P-A J} \times F_{G R}$ is the moment due to the ground reaction force in relation to the ankle joint centre, and the sum $M_{G R-A J}+M_{\text {free }}$ is the total external moment $M_{E x t}$ applied to the foot (see Figure 1, on the right). It clearly appears that the power transmitted to the shank is the sum of a translational power $\left(P_{\text {transl }}=F_{G R} \cdot V_{A J}\right)$ and a rotational power $\left(P_{\text {rot }}=M_{E x t} \cdot \omega_{\text {sh }}\right)$. In this operation the presence of the foot is not considered. In fact, the mass of foot is relatively small and the foot is almost still during the stance phase, so that its weight and inertia forces are negligible. Although the foot is not analysed, the power computed in this way intrinsically is affected by the foot deformation, and so we called this method the 'Deformable Foot (DF)' method. Instead, the traditional method of computing the ankle joint power, that we will call the 'Ankle Joint (AJ)' method, considers the foot as a rigid beam connected to the shank by an ideal rotational joint (see Figure 1, on the left). The power in this case is computed as:

$$
P_{A J}=M_{A J} \cdot \omega_{A J}
$$

where $P_{A J}$ is the power at the ankle joint; $M_{A J}$ is the ankle joint internal moment; $\omega_{A J}$ is the angular velocity of the relative rotation between foot and shank. In theory the ankle joint power should be transferred to the shank and it should be the same as the one obtained by the DF method, but this would not happen if the foot does not behave as a rigid beam. The differences between the two methods were thus analyzed.

Once the powers were calculated with the two methods, the energy flow was computed in both cases by integrating the power over time:

$$
E=\int P(t) d t
$$

Positive values of the energy mean that the foot is producing work and the power is transmitted to the tibia while negative values represent the absorption of energy by the foot because of the transmission of power from the tibia to the foot.

\section{Experimental protocol implementation}

Retroreflective markers (spherical, $15 \mathrm{~mm}$ diameter) were positioned on the medial and lateral malleoli and on medial and lateral femoral epicondyles of both limbs of our experimental subjects. 
These were intended for the identification of the longitudinal axis of the shank and to define a local reference system of Cartesian axes. Two additional markers were put on the first and fifth metatarsal heads in order to identify the foot longitudinal axis and its local reference frame. These last two markers were only required for the computation of the foot-shank rotational velocity, as needed by the more traditional AJ method and were not required by the DF method.

Kinematic and dynamic data were collected by means of a motion analysis system (Smart-E, BTS, Italy) with 6 cameras (sampling frequency of $120 \mathrm{~Hz}$ ), and a force platform (sampling frequency of $960 \mathrm{~Hz}$, Kistler, 9286AA, Switzerland). The centre of the ankle joint (A) was defined as the midpoint of the markers placed on the medial and lateral malleoli; the longitudinal axis of the shank $\left(Z_{s h}\right)$ was identified as the line connecting the ankle joint centre to the midpoint between the medial and lateral femoral epicondyles (point $\mathrm{P}$ ); the longitudinal axis of the foot $\left(Z_{\text {foot }}\right)$ was defined as the line which connects the midpoint of the markers on the first and fifth metatarsal heads to the ankle joint centre. The posterior-anterior axis of the shank $\left(Y_{s h}\right)$ was obtained as the perpendicular to the plane defined by the markers on the medial and lateral malleoli and the point $P$. The medial-lateral axis of the shank ( $X_{s h}$, exiting to the right) was then obtained by the cross product of $Y_{s h}$ and $Z_{s h}$. The angular velocity of the shank $\left(\omega_{s h}\right)$ was obtained by first defining the three Euler angles of the shank reference frame in relation to the absolute reference frame (the laboratory frame). Named $\theta, \chi, \phi$ the nutation, precession, and rotation angles respectively, the three components of the angular velocity in the shank reference frame are defined by:

$$
\begin{gathered}
\omega_{x_{-} s h}=\dot{\chi} \sin \theta \sin \phi+\dot{\theta} \cos \phi \\
\omega_{y_{-} s h}=\dot{\chi} \sin \theta \cos \phi-\dot{\theta} \sin \phi \\
\omega_{z_{-} s h}=\dot{\chi} \cos \theta+\dot{\phi}
\end{gathered}
$$

And the absolute components of the angular velocity were obtained by a rotation transformation:

$$
\omega_{s h}={ }_{A \prime}^{A}\left[R_{s h}\right] \omega_{-} s h
$$

Where ${ }_{A \prime}^{A}\left[R_{S h}\right]$ is the rotation matrix from the shank reference system $\mathrm{A}^{\prime}$ to the laboratory reference system $A ; \omega_{s}$ sh is the angular velocity vector in the shank reference system $A^{\prime}$ (whose components are $\left.\omega_{x_{-} s h}, \omega_{y_{-} s h}, \omega_{z_{-} s h}\right)$.

As to the AJ method, the angular velocity of foot in relation to shank was computed assuming that the flexion/extension axis was perpendicular to the plane defined by the longitudinal axes of foot and shank. The orientation of the vector $\omega_{A J}$ was thus obtained by the cross product of the two axes, and the relative angle was computed as:

$$
\alpha_{A J}=\operatorname{arcos}\left(\frac{Z_{\text {foot }} \cdot Z_{\text {sh }}}{\left|Z_{\text {foot }} \cdot Z_{\text {sh }}\right|}\right)
$$

The modulus of the angular velocity was obtained by computing the derivative in time of the relative angle: $\omega_{A J}=\alpha_{A J}$

\section{Participants and walking conditions}

We enrolled 18 healthy subjects: 9 males and 9 females (mean age: $26 \pm 2$ years). We excluded from the analysis subjects with orthopedic pathologies, major orthopedic surgery or motor disorders. The 
155 Ethical Committee of our Institution authorized the investigation and the participants signed an 156 informed consent which could be revoked at any moment by the subjects.

157 All participants were asked to walk in two different conditions: barefoot and with sport shoes. While 158 walking barefoot they performed the task at three different velocities: slow, normal and fast (the 159 speed was self-selected by the subject itself); in shod conditions they walked at natural speed. The 160 worn shoes were common sport shoes that each subject used currently in his/her daily life. In

161

162

163 164 165 166 addition to these walking trials, 4 female subjects (mean age: $27 \pm 1$ years) were asked to walk also in high heel shoes (average height $8 \pm 2 \mathrm{~cm} \mathrm{SD}$ ) at their preferred speed. For the analysis, we considered only the trials in which the subject stepped on the force platform with a single foot, without artificially lengthening or shortening the step. Trials with truncated or double steps on the force plate were excluded from further analysis. For each subject and walking condition, we collected five trials for each limb.

\section{Parameters of interest and Statistical Analysis}

We calculated the mean power and energy curves for each subject in each different walking condition with the DF and AJ methods. Power and energy were normalized to the mass of the subject. We evaluated the maximum value of the power, the first minimum within the $20 \%$ of the stance phase and the second minimum during the last $70 \%$ of the stance phase. We also calculated the final value of energy, the absorbed and the produced energy. For each parameter and condition, we calculated the Mahalanobis distance between the observations and removed the outlier values. We tested the distribution of each parameter for normality by the Shapiro-Wilk test. We transformed the parameters with non-normal distribution by the logarithmic transformation and tested again the transformed parameter for normality. The statistical comparison across the two methods was conducted by means of a matched pairs analysis performed with the JMP statistical package (JMP 13, SAS Institute Inc., Cary, NC, USA) with p-value set at 0.05 .

\section{Results}

\section{Barefoot Condition}

The comparison between the average curves of power and energy obtained by the two methods revealed significant differences. The most striking diversity was found during the load response phase (0-20\% of the gait cycle), during which the DF method showed a consistent negative peak of power absorption, barely visible with the AJ method (Figure 2, panel A). The energy curve was consequently affected and exhibited a sort of downward translation in this phase. Also, the maximum power value calculated with the DF method was significantly lower with respect to the one calculated with the AJ method (Figure 2, panel A and Table 1). This difference resulted in a lower final energy value calculated with the DF method with respect to the AJ method. Also, the total energy absorbed during the first part of the stance phase (0-30\%) and produced during the terminal stance (70-100\%) was statistically different across the two methods (Figure 2, panel B). These considerations apply to all gait velocities and walking conditions (Table 1).

The final energy value calculated with the DF method was averagely negative at all gait velocity except for the fast condition, in which it reached a positive value. Conversely, the final energy value resulted from the AJ method was always positive. As expected, all the parameters of both methods showed higher values as velocity increased. 


\section{Shod Condition}

The power and energy curves calculated during walking with sports shoes and in the barefoot condition showed similar morphology and values (Figure 3, panels A and B, and Table 2). The same significant differences between the two methods were obtained in the barefoot and in the sports shoes conditions. During walking in high heels, the power and energy curves were characterized by peculiar features not present in the other walking conditions (Figure 3, panels C and D). In particular, during the load acceptance phase the DF method revealed two peaks of absorption instead of the one visible with the AJ method. Furthermore, the maximum value of power and the produced energy were lower with respect to the other conditions with both methods. Walking in high heels was also the only condition in which the average final energy value was negative with both methods. All the evaluated parameters were different across the two methods also in high heels condition, except for the Second Min Power and Energy Produced.

\section{Discussion}

In this study we compared the AJ method and the DF method for power and energy calculation during gait, investigating their applicability in a clinical setup. Our results revealed the limitations of the AJ method, showing that modelling the foot as a rigid segment might lead to an incorrect evaluation of power and energy during gait. The DF method, instead, intrinsically takes into account foot deformations, particularly of the rearfoot, which play a major role in power absorption and production during walking. In addition, this method can be implemented with a simpler markers protocol, which might be an advantage for clinical applications.

In particular, the DF method reveals interesting features of the energy associated to foot deformation that don't appear with the AJ method. The first negative peak of the power curve represents the absorbed power after the heel contact and it is strictly related to the deformation of the foot during the load acceptance phase. This difference across the two methods became striking for the high heels condition. With the DF method we found a double-peaked curve, likely due to the gap between the heel and the forefoot of the shoe, which split the load acceptance into two parts (first the contact of the heel and then the contact of the forefoot). The assumptions underlying the foot model of the AJ method prevented to capture these dynamics. The positive peak of power obtained with the AJ method was always higher with respect to the DF method. This is because with the AJ method the power absorption due to the deformation of the foot structure is neglected. The consequence could be that a possible mechanism of elastic energy recovering is underestimated. Considering that the gastrocnemius and the soleus are the muscles playing a major role during the propulsive phase, it can be also important to correctly estimate the propulsive capacity of these plantar-flexor muscles.

A correct estimation of power and energy absorbed and produced by the foot is crucial for some pathological conditions. In the case of patients with cerebral palsy, for example, a common feature is the equinus foot. This anomaly is characterized by enhanced plantarflexion which leads to toewalking and can be associated to structural deformities, spasticity of the triceps surae muscles, contractures. The quantification of the absorption and production of energy during stance phase, which is made possible by the DF method, is fundamental to understand the mechanical conditions 
238 of the foot and to plan for proper interventions. This information can be obtained without any 239 complication of the data acquisition protocol.

240 There are also many orthopaedic pathologies, such as flatfoot, arthritis, tendonitis which affect the 241 feet and need a reliable quantitative measure of power for a correct treatment planning and 242 assessment. In the area of prosthetics and orthotics the energy parameters are fundamental to 243 assess the effectiveness of these devices. For example, prosthetic feet for lower limb amputations 244 are currently based on the concept of energy store and restitution (ESR devices). As the name 245 suggests, these prosthetic feet absorb energy in the loading phase and return it during the push off 246 phase. Therefore, energy parameters are fundamental to determine the efficiency of the system 247 and the effects of the aesthetic cover and of the shoes.

248 One of the strong points of the DF method is that it does not require the application of a complex 249 foot marker protocol [1], [2] in that it takes into account foot deformation just by considering the 250 rotational and translational power transmitted to the shank. The advantage of not placing many 251 markers on the foot is remarkable especially when considering pathological subjects with bone 252 deformities or children, in which markers placement might be particularly complex. Furthermore, 253 this method might be applicable to shod walking and to prosthetic subjects as well.

\section{Conflicts of interest statement}

On behalf of all authors, the corresponding author states that there is no conflict of interest.

\section{Author contributions}

VF and CAF designed the experiment. CAF recruited the participants. VF and LH acquired the data. VF, LH and CP analyzed the data. VF, CP and CAF wrote the article. CP and CAF reviewed the manuscript. 


\section{References}

[1] Simon, J., Doederlein, L., McIntosh, A.S., Metaxiotis, D., Bock, H.G., Wolf, S.I., 2006. The Heidelberg foot measurement method: Development, description and assessment. Gait Posture 23, 411-424. https://doi.org/10.1016/j.gaitpost.2005.07.003

[2] Carson, M.C., Harrington, M.E., Thompson, N., O'Connor, J.J., Theologis, T.N., 2001. Kinematic analysis of a multi-segment foot model for research and clinical applications: a repeatability analysis. J. Biomech. 34, 1299-1307. https://doi.org/10.1016/S00219290(01)00101-4

[3] Baker, R., Robb, J., 2006. Foot models for clinical gait analysis. Gait Posture 23, 399-400. https://doi.org/10.1016/j.gaitpost.2006.03.005

[4] Cappozzo, A., 1984. Gait analysis methodology. Hum Mov Sci 3. https://doi.org/10.1016/0167-9457(84)90004-6

[5] Davis, R.B., Õunpuu, S., Tyburski, D., Gage, J.R., 1991. A gait analysis data collection and reduction technique. Hum Mov Sci 10. https://doi.org/10.1016/0167-9457(91)90046-Z

[6] Frigo, C., Rabuffetti, M., Kerrigan, D.C., Deming, L.C., Pedotti, A., 1998. Functionally oriented and clinically feasible quantitative gait analysis method. Med. Biol. Eng. Comput. 36, 179185. https://doi.org/10.1007/BF02510740

[7] Kadaba, M.P., Ramakrishnan, H.K., Wooten, M.E., Gainey, J., Gorton, G., Cochran, G.V.B., 1989. Repeatability of kinematic, kinetic, and EMG data in normal adult gait. J Orthop Res 7. https://doi.org/10.1002/jor.1100070611

[8] Winter, D.A., 2009. Biomechanics and motor control of human movement. 4th ed. Wiley.

[9] Whittle, M.W., 2014. Gait Analysis: An Introduction. Butterworth--Heinemann.

[10] MacWilliams, B.A., Cowley, M., Nicholson, D.E., 2003. Foot kinematics and kinetics during adolescent gait. Gait Posture 17, 214-224. https://doi.org/10.1016/S0966-6362(02)00103-0

[11] Zelik, K.E., Honert, E.C., 2018. Ankle and foot power in gait analysis: Implications for science, technology and clinical assessment. J. Biomech. 75, 1-12. https://doi.org/10.1016/j.jbiomech.2018.04.017

[12] Takahashi, K.Z., Stanhope, S.J., 2013. Mechanical energy profiles of the combined ankle-foot system in normal gait: Insights for prosthetic designs. Gait Posture 38, 818-823. https://doi.org/10.1016/j.gaitpost.2013.04.002

[13] Takahashi, K.Z., Worster, K., Bruening, D.A., 2017. Energy neutral: the human foot and ankle subsections combine to produce near zero net mechanical work during walking. Sci. Rep. 7, 15404. https://doi.org/10.1038/s41598-017-15218-7 
Tables:

\begin{tabular}{|c|c|c|c|c|c|c|}
\hline & \multicolumn{2}{|c|}{ SLOW } & \multicolumn{2}{|c|}{ NORMAL } & \multicolumn{2}{|c|}{ FAST } \\
\hline & AJ & DF & AJ & DF & AJ & DF \\
\hline Power peak [W/Kg] & $\begin{array}{c}2.19 \\
(0.64)\end{array}$ & $\begin{array}{l}1.70 \\
(0.51)\end{array}$ & $\begin{array}{c}3.15 \\
(0.68)\end{array}$ & $\begin{array}{l}2.44 \\
(0.56)\end{array}$ & $\begin{array}{c}4.21 \\
(0.84)\end{array}$ & $\begin{array}{c}3.64 \\
(0.78)\end{array}$ \\
\hline First Min Power $[\mathrm{W} / \mathrm{Kg}]$ & $\begin{array}{l}-0.18 \\
(0.07)\end{array}$ & $\begin{array}{l}-0.65 \\
(0.29)\end{array}$ & $\begin{array}{l}-0.23 \\
(0.09)\end{array}$ & $\begin{array}{l}-1.16 \\
(0.47)\end{array}$ & $\begin{array}{l}-0.47 \\
(0.22)\end{array}$ & $\begin{array}{l}-2.60 \\
(0.88)\end{array}$ \\
\hline $\begin{array}{l}\text { Second Min Power } \\
\qquad[\mathrm{W} / \mathrm{Kg}]\end{array}$ & $\begin{array}{l}-0.70 \\
(0.24)\end{array}$ & $\begin{array}{l}-0.82 \\
(0.29)\end{array}$ & $\begin{array}{l}-0.79 \\
(0.30)\end{array}$ & $\begin{array}{l}-0.94 \\
(0.36)\end{array}$ & $\begin{array}{l}-0.70 \\
(0.38)\end{array}$ & $\begin{array}{l}-0.93 \\
(0.48)\end{array}$ \\
\hline Final energy value $[\mathrm{J} / \mathrm{Kg}]$ & $\begin{array}{c}0.06 \\
(0.05)\end{array}$ & $\begin{array}{l}-0.05 \\
(0.07)\end{array}$ & $\begin{array}{c}0.10 \\
(0.06)\end{array}$ & $\begin{array}{l}-0.03 \\
(0.07)\end{array}$ & $\begin{array}{c}0.21 \\
(0.09)\end{array}$ & $\begin{array}{l}0.045 \\
(0.10)\end{array}$ \\
\hline Energy Produced [J/Kg] & $\begin{array}{c}0.22 \\
(0.06)\end{array}$ & $\begin{array}{c}0.18 \\
(0.05)\end{array}$ & $\begin{array}{c}0.24 \\
(0.05)\end{array}$ & $\begin{array}{c}0.19 \\
(0.04)\end{array}$ & $\begin{array}{c}0.30 \\
(0.07)\end{array}$ & $\begin{array}{c}0.24 \\
(0.06)\end{array}$ \\
\hline Energy Absorbed [J/Kg] & $\begin{array}{l}-0.16 \\
(0.05)\end{array}$ & $\begin{array}{l}-0.23 \\
(0.06)\end{array}$ & $\begin{array}{l}-0.14 \\
(0.05)\end{array}$ & $\begin{array}{l}-0.22 \\
(0.07)\end{array}$ & $\begin{array}{l}-0.08 \\
(0.05)\end{array}$ & $\begin{array}{l}-0.20 \\
(0.07)\end{array}$ \\
\hline
\end{tabular}

Table 1 Values of the main parameters of power and energy curves during walking at three different velocities; all comparisons across the two methods were statistically significant $(p<0.05)$. Values are shown as mean (standard deviation). 


\begin{tabular}{|c|c|c|c|c|}
\hline & \multicolumn{2}{|c|}{ SPORTS SHOES } & \multicolumn{2}{|c|}{ HIGH HEELS } \\
\hline & AJ & DF & AJ & DF \\
\hline Power peak $[\mathrm{W} / \mathrm{Kg}]$ & $\begin{array}{c}3.09 \\
(0.69)\end{array}$ & $\begin{array}{c}2.49 \\
(0.57)\end{array}$ & $\begin{array}{c}1.37 \\
(0.60)\end{array}$ & $\begin{array}{c}1.18 \\
(0.43)\end{array}$ \\
\hline $\begin{array}{l}\text { First Min Power } \\
\qquad[\mathrm{W} / \mathrm{Kg}]\end{array}$ & $\begin{array}{l}-0.30 \\
(0.09)\end{array}$ & $\begin{array}{l}-1.08 \\
(0.44)\end{array}$ & $\begin{array}{l}-0.22 \\
(0.09)\end{array}$ & $\begin{array}{l}-1.31 \\
(0.61)\end{array}$ \\
\hline $\begin{array}{l}\text { Second Min Power } \\
{[\mathrm{W} / \mathrm{Kg}]}\end{array}$ & $\begin{array}{l}-0.78 \\
(0.33)\end{array}$ & $\begin{array}{l}-0.95 \\
(0.39)\end{array}$ & $\begin{array}{l}-0.98 \\
(0.25)\end{array}$ & $\begin{array}{l}-1.08 \\
(0.40)\end{array}$ \\
\hline $\begin{array}{l}\text { Final energy value } \\
\qquad[\mathrm{J} / \mathrm{Kg}]\end{array}$ & $\begin{array}{c}0.09 \\
(0.06)\end{array}$ & $\begin{array}{l}-0.03 \\
(0.75)\end{array}$ & $\begin{array}{l}-0.02 \\
(0.05)\end{array}$ & $\begin{array}{l}-0.09 \\
(0.06)\end{array}$ \\
\hline $\begin{array}{l}\text { Energy Produced } \\
{[\mathrm{J} / \mathrm{Kg}]}\end{array}$ & $\begin{array}{c}0.24 \\
(0.05)\end{array}$ & $\begin{array}{c}0.19 \\
(0.04)\end{array}$ & $\begin{array}{c}0.08 \\
(0.04)\end{array}$ & $\begin{array}{c}0.08 \\
(0.03)\end{array}$ \\
\hline $\begin{array}{c}\text { Energy Absorbed } \\
{[\mathrm{J} / \mathrm{Kg}]}\end{array}$ & $\begin{array}{l}-0.15 \\
(0.05)\end{array}$ & $\begin{array}{l}-0.23 \\
(0.07)\end{array}$ & $\begin{array}{l}-0.11 \\
(0.03)\end{array}$ & $\begin{array}{l}-0.17 \\
(0.05)\end{array}$ \\
\hline
\end{tabular}

Table 2 Values of the main parameters of power and energy curves during walking in sport shoes and high heels; all comparisons across the two methods were statistically significant $(p<0.05)$ except for the second min power and the energy produced during walking with high heels. Values are shown as mean (standard deviation). 
Figures:

306 Figure 1 Models of power computation. Left: the ankle joint (AJ) method; right: the deformable foot 307 (DF) method (see the Methods section for details). $F_{G R}=$ ground reaction force; $M_{A J}=$ moment of 308 the external forces applied at the ankle joint; $\omega_{A J}=$ angular velocity of the ankle joint; $P_{A J}=$ power at the ankle joint; $\mathrm{M}_{\mathrm{EXT}}=$ external moment applied to the shank; $\omega_{S H}=$ angular velocity of the shank; $\mathrm{V}_{\mathrm{AJ}}=$ linear velocity of the center of the ankle joint; $\mathrm{P}_{\mathrm{GR}-\mathrm{SH}}=$ power exchanged between ground and 311 shank.

Figure 2 Average curves (+/- 1 standard deviation) of Power (A) and Energy (B) obtained at normal velocity from all subjects in barefoot condition (red line: DF method; black line: AJ method)

Figure 3 Average curves (+/- 1 standard deviation) of Power $(A, C)$ and Energy (B,D) obtained at normal velocity from all subjects walking in sports shoes $(A, B)$ and high heels $(C, D)$ (red line: DF method; black line: AJ 